\title{
Guest Editorial: Cross-Layer Design for Future Generation Wireless Networks
}

\author{
Yuh-Shyan Chen • Athanasios V. Vasilakos • \\ Chien-Chung Shen
}

Published online: 26 June 2009

(C) Springer Science+Business Media, LLC. 2009

With the rapid development of wireless technologies, the future generation wireless networks have more challenges for providing reliable and high quality end-to-end performance in wireless multimedia communications. A cross-layer design has become a popular topic in which more and more researchers are engaged. That leads to a lot of cross-layer design proposals for wireless networks. This special issue aims to collect and foster stateof-the-art research pertaining to cross-layer design in the area of future generation wireless networks. The emphasis of this special issue will be on cross-layer protocol interactions and design of cross-layer optimized techniques.

The accepted fourteen papers are grouped into six main themes. The first set of papers deals with the cross-layer energy-efficient and power control mechanisms. The second set reports the cross-layer routing protocols. The third set of papers investigates the TCP improvement schemes using cross-layer design. The fourth set of papers describes the cross-layer multimedia schemes. The fifth set of papers displays the cross-layer QoS scheduling schemes. The final set of papers discusses the cross-layer design in IEEE 802.16 networks.

In the first set of papers, the cross-layer energy-efficient design and power control mechanisms are reported. In the first paper, Li-Chun Wang, Jane-Hwa Huang, Anderson Chen, and Chung-Ju Chang, proposed the cross-layer analysis of joint rate and power adaptation in Nakagami fading channels with multiple-user contention. The authors proposed channeldriven rate and power adaptation (CDRPA) schemes and develop a physical (PHY)/medium

Y.-S. Chen $(\varangle)$

National Taipei University, Taipei, Taiwan, ROC

e-mail: yschen@mail.ntpu.edu.tw

A. V. Vasilakos

University of Western Macedonia, Kozani, Greece

e-mail: vasilako@ath.forthnet.gr

C.-C. Shen

University of Delaware, Newark, DE, USA

e-mail: cshen@cis.udel.edu 
access control (MAC) cross-layer analytical method incorporating the impacts of Nakagami fading channel and the carrier sense multiple access (CSMA) MAC protocol. In the second paper, Konstantinos P. Tsoukatos, Maria-Pinelopi Chrisanthopoulou and Apostolos Apostolaras, considered the backlog-driven power control and antenna beamforming as a means to maximize network throughput in a wireless uplink, where a base station receives with an antenna array from single-antenna wireless users. The authors introduce a cross-layer, alternating maximization algorithm searching for the optimal solution, and a variant of the algorithm with reduced complexity. In the third paper, Chia-Hung Tsai, Tsu-Wen Hsu, MengShiuan Pan, and Yu-Chee Tseng developed a cross-layer, energy-efficient design for queryprocessing protocol to simultaneously support multiple continuous queries in a wireless sensor network. This work interested in applying the quorum-based power-saving protocols, which have the advantage of not relying on any time synchronization among sensor nodes, to the continuous query-processing problem. This cross-layer design emphasizes on increasing the overlapping of query paths for energy efficiency.

The second part contains papers that report the cross-layer routing protocols. In the fourth paper, G. Kannan, Yangcheng Huang, Saleem Bhatti, S. N. Merchant, and U. B. Desai proposed a cross layer routing protocol for a code division multiple access (CDMA) multihop cellular networks. Multiple constraints are imposed on intermediate relay node selection and end-to-end path selection. In the fifth paper, Ghada A. Al-Mashaqbeh, Jamal N. Al-Karaki, and Sameer M. Bataineh developed a cross-layer based routing framework for multi-interface/multi-channel WMNs, called Cross-Layer Enhanced and Adaptive Routing (CLEAR) to efficiently utilize the advantages of both multi-radio/multi-channel WMNs and cross-layer design.

The third part reports the TCP improvement schemes using cross-layer design. In the sixth paper, Dionysia K. Petraki, Markos P. Anastasopoulos, Athanasios V. Vasilakos, and Panayotis G. Cottis, developed a TCP-aware call admission control in high altitude platforms using cross-layer design. A new cross-layer design is proposed employing the pre-dictability of rain faded channels to guarantee QoS requirements in High Altitude Platform (HAP) networks. In the seventh paper, Juho Ha, Eun-Chan Park, Kyung-Joon Park, and Chong-Ho Choi developed a cross-layer dual queue approach for improving TCP fairness in infrastructure WLANs. They focus on the fairness problem between TCP uplink and downlink flows in infrastructure WLANs from the cross-layer perspective.

The fourth part reports the cross-layer multimedia schemes. In the eighth paper, Hyungkeuk Lee, Suyoung Park, and Sanghoon Lee developed a dynamic session control over IMS for cross-layer optimization of multi-stream video. In the ninth paper, Syed Ali Khayam and Hayder Radha developed a comparison of conventional and cross-layer multimedia transport schemes for wireless networks. Three competing schemes have been proposed for multimedia transport over broadband wireless channels: (a) traditional UDP, (b) semicross-layer UDP-Lite, and (c) cross-layer header estimation.

The fifth part reports the cross-layer QoS scheduling schemes. In the tenth paper, Niar Zorba, Ana I. P'erez-Neira, Andreas Foglar, and Christos Verikoukis developed a cross layer QoS guarantees in multiuser WLAN systems. A cross-layer based dynamically tuned queue length scheduler is presented for the downlink of multiuser WLAN systems with heterogeneous traffic requirements. In the eleventh paper, Yuedong Xu, XiaoxinWu, and John C.S. Lui developed a cross-layer QoS scheduling for layered multicast streaming in OFDMA wireless networks. This work investigates the cross-layer subchannel assignment mechanism of multicast streaming services in the emerging WiMax/802.16e systems, where each multimedia stream is composed of a basic layer and an enhancement layer. In the twelfth paper, Chi Harold Liu, Athanasios Gkelias, Yun Hou, and Kin K. Leung developed a cross-layer design 
for QoS in wireless mesh networks. The authors proposed a unified framework that exploits the physical channel properties and multi-user diversity gain of WMNs and by performing intelligent route selection and connection admission control provides both QoS and GoS to a variety of underlying applications.

The sixth part reports the cross-layer designs in IEEE 802.16 networks. In the thirteenth paper, Jian Zhang, Honglin Hu, Lu Rong, and Hsiao-Hwa Chen developed a cross-layer scheduling algorithms for IEEE 802.16 based wireless mesh networks. The authors discuss the issues on how to satisfy a set of bandwidth requests in IEEE 802.16 WMNs using minimal radio resources. In the last paper, Fei Yin, Guy Pujolle, Jong Hyuk Park, and Han-Chieh Chao developed a performance optimization with efficient polling mechanism in IEEE 802.16 networks with cross-layer consideration.

We thank all authors for their outstanding contributions. We selected 14 papers for inclusion in this special issue from 58 submissions. These accepted contributions came from different parts of the world including: Taiwan (4), Greece (2), United States (1), United Kingdom (1), China (1), Spain (1), India (1), Korea (1), Pakistan (1), and Jordan (1).

We would also like to thank Professor Ramjee Prasad, the Editor-in-Chief of the Wireless Personal Communications for his kind encouragements and invaluable support during the preparation of this special issue. We express our deepest gratitude to all the anonymous reviewers who devoted much of their precious time reviewing all the papers. Their timely reviews greatly helped us select the best papers included in this special issue.

Finally, we hope you will enjoy reading this selection of papers as we did and you will find this issue informative and helpful in keeping yourselves up-to-date in the fast changing field of 'cross-layer' design for future generation wireless networks.

\section{Author Biographies}

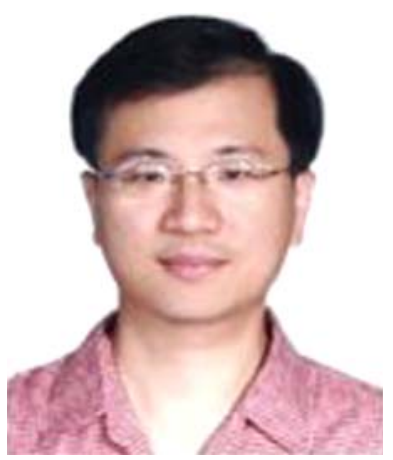

Yuh-Shyan Chen received the B.S. degree in Computer Science from Tamkang University, Taiwan, ROC, in June 1988 and the M.S. and Ph.D. degrees in Computer Science and Information Engineering from the National Central University, Taiwan, ROC, in June 1991 and January 1996, respectively. He joined the faculty of Department of Computer Science and Information Engineering at Chung-Hua University, Taiwan, ROC, as an associate professor in February 1996. He joined the Department of Statistic, National Taipei University in August 2000, and joined the Department of Computer Science and Information Engineering, National Chung Cheng University in August 2002. Since 2006, he has been a Professor at the Department of Computer Science and Information Engineering, National Taipei University, Taiwan. Prof. Chen is now serving as chair of Institute of Communication Engineering, National Taipei University, Taiwan, ROC, and Vice Chair of Task Force on "Telecommunications" of Intelligent Systems Applications Technical Committee, IEEE Computational Intelligence Society. Prof. Chen served as Editor-in-Chief of International Journal of Ad Hoc and Ubiquitous Computing (SCIE), Editorial Board of Telecommunication System Journal (SCIE), EURASIP Journal on Wireless Communications and Networking (SCIE), and Mobile Information Systems (SCIE). He served as Guest Editor of ACM/Springer Mobile Networks and Applications (MONET), Telecommunication Systems, Wireless Communications and Mobile Computing, EURASIP Journal on Wireless Communications and Networking, The Computer Journal, Wireless Personal Communications, International Journal of Communication Systems, and IET Communications. His paper wins the 2001 IEEE 15th ICOIN-15 Best Paper Award. Prof. Chen was a recipient of the 2005 Young Scholar Research Award, National Chung Cheng University, ROC. His recent research topics include wireless communications, mobile computing, and next-generation personal communication system. Dr. Chen is a member of the IEEE Communication Society and Phi Tau Phi Society. 


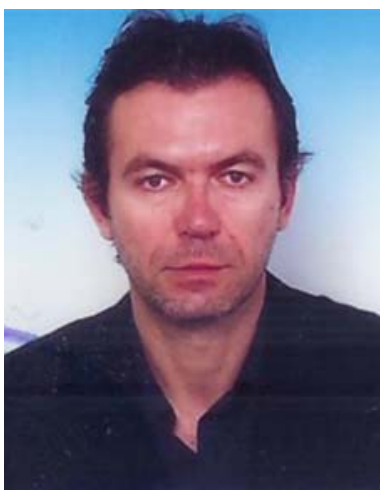

Athanasios V. Vasilakos is currently Professor at the Department of Computer and Telecommunications Engineering, University of Western Macedonia, Greece, and Visiting Professor at the Graduate Programme of the Department of Electrical and Computer Engineering, National Technical University of Athens (NTUA). He is coauthor (with W. Pedrycz) of the books Computational Intelligence in Telecommunications Networks (CRC Press, USA, 2001), Ambient Intelligence, Wireless Networking, Ubiquitous Computing (Artech House, USA, 2006), coauthor (with M. Parashar, S. Karnouskos, W. Pedrycz) Autonomic Communications (Springer, to appear), Arts and Technologies (MIT Press, to appear), coauthor (with Yan Zhang, Thrasyvoulos Spyropoulos) Delay Tolerant Networking (CRC Press, to appear), coauthor (with M. Anastasopoulos) Game Theory in Communication Systems (IGI Inc., USA, to appear). He has published more than 200 articles in top international journals (i.e IEEE/ACM Transactions on Networking, IEEE T-Information Theory, IEEE JSAC, IEEE Transactions on Wireless Communications, IEEE Transactions on Neural Networks, IEEE Transactions on Systems, Man, \& Cybernetics, IEEE T-ITB, IEEE T-CIAIG, etc.) and conferences. He is the Editor-in-Chief of the Inderscience Publishers journals: International Journal of Adaptive and Autonomous Communications Systems (IJAACS), International Journal of Arts and Technology (IJART). He was or he is at the editorial board of more than 20 international journals including: IEEE Communications Magazine (1999-2002 \& 2008-), IEEE Transactions on Systems, Man and Cybernetics (TSMC, Part B, 2007-), IEEE Transactions on Wireless Communications (invited), IEEE Transactions on Information Theory in Biomedicine (TITB, 2009-) etc. He chairs several conferences i.e., ACM IWCMC'09, ICST/ACM Autonomics 2009. He is chairman of the Telecommunications Task Force of the Intelligent Systems Applications Technical Committee (ISATC) of the IEEE Computational Intelligence Society (CIS). Senior Deputy Secretary-General and fellow member of ISIBM www.isibm.org (International Society of Intelligent Biological Medicine (ISIBM). He is member of the IEEE and ACM.

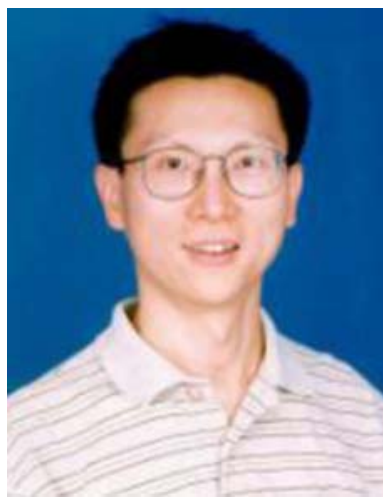

Chien-Chung Shen received his B.S. and M.S. degrees from National Chiao Tung University, Taiwan, and his Ph.D. degree from UCLA, all in computer science. He was a research scientist at Bellcore (now Telcordia) Applied Research working on control and management of broadband networks. He is now an associate professor in the Department of Computer and Information Sciences of the University of Delaware, USA. His research interests include ad hoc, sensor, and underwater networks, dynamic spectrum management, control and management of broadband networks, distributed object and peer-topeer computing, and simulation. He is a recipient of NSF CAREER Award, and his research has been supported by US NSF, Army, Navy, NASA, RAND, and industrial companies. He is a member of ACM and IEEE. 